\section{Sara Bigardi}

Università di Verona sarabigardi@gmail.com

\author{
Il Prologo di «Persona y democracia»: \\ la coscienza storica di Maria Zambrano \\ The Prologue to "Persona y democracia": \\ Maria Zambrano's historical \\ consciousness
}

\begin{tabular}{|c|c|c|}
\hline & Resumen & Abstract \\
\hline \multirow{2}{*}{$\begin{array}{l}\text { Recepción: } 2 \text { I de septiembre de } 2018 \\
\text { Aceptación: } 22 \text { de octubre de } 2018\end{array}$} & En este artículo, a partir del & In this paper, based on the prolo- \\
\hline & Prólogo de 1987 de Persona y & gue to Persona y democracia (1987 \\
\hline \multirow[t]{8}{*}{ Aurora n. ${ }^{\circ}$ 20, 2019, págs. 6-I 2} & democracia, profundizo en algunas & edition), I go into depth on some \\
\hline & $\begin{array}{l}\text { palabras clave no para ofrecer una } \\
\text { visión global del texto, sino una }\end{array}$ & $\begin{array}{l}\text { key concepts not to illustrate a } \\
\text { global vision of the text, but rather }\end{array}$ \\
\hline & $\begin{array}{l}\text { argumentación que tenga en cuenta } \\
\text { sustancialmente dos cuestiones: }\end{array}$ & $\begin{array}{l}\text { to present an argument that takes } \\
\text { into account two important issues: }\end{array}$ \\
\hline & $\begin{array}{l}\text { la capacidad que tiene Zambrano } \\
\text { de estar presente en el tiempo, }\end{array}$ & $\begin{array}{l}\text { Zambrano's ability to be present in } \\
\text { time and to clearly read reality, and }\end{array}$ \\
\hline & leyendo la realidad sin abstraccio- & how certain of her intuitions are \\
\hline & $\begin{array}{l}\text { nes, y como ciertas intuiciones son } \\
\text { germinativas para nuestro presente. }\end{array}$ & germinative for our present. \\
\hline & Palabras clave & Keywords \\
\hline & $\begin{array}{l}\text { Política, historia, crisis, conciencia } \\
\text { histórica, tiempo }\end{array}$ & $\begin{array}{l}\text { Politics, history, crisis, historical } \\
\text { conscience, time }\end{array}$ \\
\hline
\end{tabular}

Per comprendere la storia nella sua interezza, nel suo intimo funzionamento, bisogna ammettere l'incredibile, bisogna constatare l'assurdo o almeno registrarlo.

María Zambrano

I. Zambrano, M., "Prólogo», in Persona y democracia. La historia sacrificial, Madrid, Ediciones Siruela, 1996, p. I2

2. Cfr. Ortega y Gasset, J., Aurora della ragione storica, Milano, SugarCo Edizioni, 2009, p. II5.
«No hay crisis, lo que hay más que nunca es orfandad». ${ }^{\mathrm{I}}$ Questa è una frase emblematica del Prólogo, scritto nel 1987, di Persona y democracia, libro apparso "por primera vez en la isla de Puerto Rico en el año 1958 en circunstancias bien diferentes, al parecer, de las que hoy [quando scrive il Prologo] se muestran en el mundo». Certo, sono passati quasi trent'anni e un lungo esilio. Inevitabile una nuova presa di coscienza; necessaria un'altra lettura del reale, visto che la storia, parafrasando Ortega y Gasset, «è il dramma che si snoda tra l'io e le circostanze». ${ }^{2}$ 
Il Prologo è un intertempo, un tempo parziale dentro una frazione di storia, denso di spunti e orientante anche per il nostro presente. Per questo sento il desiderio, ma soprattutto il bisogno e l'urgenza, di partire da qui. Iniziare dal breve scritto introduttivo mi permette di riflettere e interrogare alcune parole e certuni sintagmi che compaiono, poi, nella prima parte di Persona y democracia.

L'idea di non trascurare il Prologo è nata per due motivi, in parte connessi. Il primo ha a che vedere con il fatto che Persona y democracia, come nota correttamente Nunzio Bombacci, «non sempre presenta un ordine espositivo perspicuo al lettore» e, mancando «un'adeguata analisi dei processi istituzionali nei quali si articola la vita politica", è difficile "accreditare a Zambrano una compiuta filosofia della politica».3 Questo, se da un lato rende problematica una disamina dell'opera nel suo complesso, dall'altro permette, invece, di soffermarsi e capire nel dettaglio, attraverso alcune parole chiave, certi frammenti di analisi proposti da Zambrano e validi anche per la contemporaneità.

Il secondo motivo riguarda il momento storico in cui viviamo. Michele Rech, in arte Zerocalcare, ${ }^{4}$ sostiene che "quel che funziona nel mercato dell'informazione e della politica non sia la complessità ma la semplificazione e la sottrazione». 5 E propone, perché ne percepisce l'urgenza, «una lettura dei fenomeni più articolata. Magari diversa dal cosiddetto discorso dominante: punti di vista, angolazioni fuori dal coro». ${ }^{6}$

Pensiero, questo, condiviso anche da alcuni giornalisti e scrittori che descrivono la società odierna come caratterizzata da una vacuità fattuale in cui, spesso, la vera comprensione e dimostrazione di ciò che succede restano sullo sfondo, lasciando spazio all'opinione corrente. ${ }^{7}$ Tutto rimane in superficie e astratto, si ragiona per grandi categorizzazioni, e l'informazione è perlopiù fatta di slogan, annunciati attraverso un linguaggio incontrollato, a tratti violento, dove i sentimenti circolanti sono risentimento, denuncia, petizioni senza soggettività e mancanza di senso storico e memoria.

Secondo il filosofo Marcel Gauchet, «il discorso sociale organizzatore, annodato attorno al complesso giuridico-tecnico-mercantile, si è svuotato della dimensione simbolica. La popolazione non vi riconosce la propria vita, visto che le indagini di opinione ne riecheggiano continuamente la denuncia». ${ }^{8}$ Se la dimensione simbolica viene svuotata, viene meno non solo la possibilità di riconoscere la propria vita, ma anche di esprimerla, ${ }^{9}$ con "esercizi di immaginazione e di visione politica», ${ }^{\circ}$ come scrive Salvatore Veca.

Tutto allora tende a farsi sociale. Questo prevalere del sociale viene analizzato da Chiara Zamboni in un articolo contenuto nel libro Femminismo fuori sesto. Un movimento che non può fermarsi. ${ }^{\text {II }}$
3. Bombacci, N., "Persona e democrazia nel pensiero di María Zambrano", in Dialegesthai 2016, url: https://mondodomani.org/dialegesthai/nbı.htm. In Zambrano c'è una visione politica anche se non compiuta, nel senso di definita. C’è un passaggio dove Zambrano dice che cosa è per lei la politica. Si trova in Orizzonte del liberalismo. «Si fa politica ogni volta che si pensa di imprimere una direzione alla vita. [...] La politica è l'attività più strettamente umana [...] è riforma, creazione $[\ldots]$ suppone una coscienza storica", Orizzonte del liberalismo, a cura e trad. it. di Donatella Cessi Montalto, Milano, Selene edizioni, 2007, pp. 79-8o-8I-83.

4. Michele Rech, in arte Zerocalcare, è un fumettista italiano.

5. Zerocalcare, «Fascismo mainstream e responsabilità mediatiche» in MicroMega. Per una sinistra illuminista, n. 3/2018, «E la stampa bellezza» - almanacco di giornalismo, pp. 195-200, cit. p. 195.

6. Ivi, cit. p. 197.

7. Cfr. Gauchet, M., «La guerra delle verità», trad. it. di Sabrina Tortorella, MicroMega. Per una sinistra illuminista, $\mathrm{n}^{\circ}$ 3/20I8, "E la stampa bellezza» - almanacco di giornalismo, pp. 67-77.

8. Gauchet, M., "La guerra delle verità», cit. p. 75 .

9. «Il lavoro da fare oggi - afferma Annarosa Buttarelli in un'intervista di Marina Terragni - è inventare un linguaggio pubblico che sappia esprimere i bisogni reali delle persone, bisogni che sono ben chiari, contrastando la retorica populista. Si tratta di un tentativo rischioso, perché in questo lavoro di invenzione si sfiora la stessa soglia del populismo. È un po' come quando il pensiero della differenza sessuale ha rischiato di essere assimilato al pensiero della destra. In buona sostanza si tratta di leggere la realtà e saperla raffigurare sulla base del pensiero dell'esperienza. Per esempio: leggere il fenomeno migratorio senza appiattire le differenze, che non sono solo quelle tra rifugiati e migranti economici. La prima differenza da considerare è quella sessuale. Noi donne siamo capaci di cogliere le differenze, gli uomini molto meno.» Intervista di Marina Terragni ad Annarosa Buttarelli pubblicata sul sito http://marinaterragni.it.

Io. «L'invito all'immaginazione politica prende le mosse dallo smascheramento e dal sospetto critico e illuministico nei confronti della falsa necessità. Prende le mosse dalla voglia di verità e veridicità. E di giustizia.» 
Salvatore Veca, "Non c’è alternativa». Falso!, Bari, Laterza, 20I4, p. XIV.

II. Zamboni, Ch., "Un movimento che si scrive passo passo", in Femminismo fuori sesto. Un movimento che non può fermarsi, Napoli, Liguori Editore, 20I7, pp. 5-22. Il paragrafo dedicato alla critica del sociale si intitola «Un fantasma si aggira tra di noi:

il sociale», pp. 9-I5.

I2. Ivi, cit. p. Io.

I3. Questa è l'idea di Hannah Arendt, cfr., Vita Activa, trad. it di Sergio Finzi,

Milano, Bompiani, 1989, p. 24.

I4. Zamboni, Ch., «Un movimento che si scrive passo passo", cit. p. I2.

15. Ibidem.

I6. Ibidem.

17. Ivi, cit. p. I5.

18. Zambrano, M., Persona y democracia, cit. p. 188.

19. Ivi, cit. p. I89.
Zamboni introduce e sviluppa la critica del sociale in riferimento al femminismo riprendendo le posizioni di Hannah Arendt e Simone Weil. Senza entrare nel merito di questa elaborazione, ciò che mi preme portare all'attenzione sono due momenti del suo ragionamento. Zamboni, partendo dall'assunto che la rappresentazione prevalente che circola nel simbolico dominante è che tutto sia sociale, "parola passe-partout per interpretare realtà molto diverse tra loro», ${ }^{12}$ individua come prima conseguenza, sulla scia di Arendt, la non distinzione tra privato e pubblico. Se il sociale è la compenetrazione tra privato-pubblico, ${ }^{13}$ con l'idea femminista che il personale è politico si apre una strada completamente diversa. Tra le diverse interpretazioni di questa idea, quella più convincente per Zamboni è che "ciò che avviene nella propria vita va letto come esperienza soggettiva in rapporto a ciò che avviene nel mondo». ${ }^{14}$ Ciò che ci capita non è solo un fatto privato, ma un modo per leggere simbolicamente il fuori e trovare pratiche per starci. «Allo stesso modo una certa visione soggettiva può trovare mediazioni giuste per diventare qualcosa di condiviso pubblicamente. In entrambi i casi quel che occorre fare di politico è proporre mediazioni linguistiche e pratiche per operare questo passaggio». ${ }^{15}$ Trovare e avere bisogno di mediazioni significa stare in una posizione di squilibrio. «Mentre nel sociale tutto circola senza apparenti squilibri». ${ }^{16}$

L'altro punto interessante affrontato da Zamboni, che riprende la posizione a cui accennavo in precedenza, è che il sociale seduce perché offre e mette a disposizione un'opinione a cui aderire e un comportamento da seguire. Entrambi ritenuti validi. "Così [chi segue i valori del sociale] non avrà da pentirsi né da scusarsi, avendo aderito alle opinioni del proprio tempo, al pensiero di tutti». ${ }^{17}$

Chi segue i valori sociali, è per Zambrano la massa, che si contraddistingue dal popolo per una espressività che disconosce «las situaciones, las circustancias y antes que nada, al interlocutor». ${ }^{18} \mathrm{Un}$ linguaggio che non si fa mediazione, che non inventa pratiche, non segnala una realtà.

Con su agobiante abuso del yo opino, del yo digo que esto es así, con su abstracción excesiva del tiempo, con el uso compensatorio de los adjetivos, el lenguaje de la masa es esquematización del lenguaje racionalista del hombre culto moderno. [...] Producto de la demagogia, la demagogia misma cristalizada. ${ }^{19}$

Per questo, procedere con un discorso globale e includente, massivo, non ha senso. È utile ed efficace, invece, restringere il campo di osservazione e andare a cogliere quelle mediazioni, soprattutto linguistiche, che stanno in rapporto con il mondo.

Il Prologo di Persona y democracia offre questa possibilità: le questioni che, abbozzando, delinea risultano significative perché si innestano in una costellazione semantica e di senso che, per le sue implica- 
Nel Prologo, la parola «crisi» non viene più utilizzata; e questo sapendo quanto Zambrano abbia lavorato sulla crisi, e non solo in Persona y democracia, ma anche in Verso un sapere dell'anima, dove è in relazione soprattutto con il sentimento di inquietudine e la perplessità. Al posto di «crisi», utilizza «orfandad», termine che va interrogato in rapporto alla storia sacrificale. La democrazia non è una questione risolta, anche se democrazia e persona rimangono le coordinate che adopera per orientare la bussola verso l'alba, al contempo descritta come la "hora más trágica del día, el momento en que la claridad aparece como herida que se abre en la oscuridad, donde todo reposa» e come luce che "anuncia y profetiza la luz que saldrá de ella misma». ${ }^{20}$ Sono parole di verità necessaria che sanno dire un preciso tempo vissuto. $\mathrm{E}$ poi ci sono parole che non si consumano perché accompagnano un'altra profonda necessità che non si perde nel tempo, un'altra verità che ci trasforma dentro e fuori. Così è efficace interrogare termini come "coscienza storica» e "tempo del risveglio".

\section{Pro-logos}

Sono due i passaggi fondamentali del Prologo. La frase che ho citato all'inizio di questo articolo li enuclea in una dimensione dialettica. ${ }^{2 \mathrm{I}}$ Il primo passaggio riguardo la democrazia e non può che essere, perlomeno all'inizio, posto in forma interrogativa. Il secondo riguarda la struttura sacrificale della storia.

A fine anni 'so, "parecía [...] abierto el camino de la democracia», di una democrazia «entrelazada con la idea de progreso», ma "¿qué se entendía entonces en el mundo occidental por democracia?, ¿qué se entiende hoy, impuesto ya el sentido de la palabra democracia? ${ }^{22}$

Ora come allora è chiaro per Zambrano che la democrazia rimane l'unico cammino "para que prosiga la llamada cultura de Occidente», ma «ni en aquel momento y todavía menos ahora, es claro, preciso y transparente el sentido real, efectivo, de ese término que filológicamente aparece tan claro». ${ }^{23}$ Filologicamente chiaro, epistemologicamente complesso. Zambrano sa quanto la democrazia abbia continuo bisogno di coscienza storica, è una parola spesso abusata, che richiede sorveglianza, attenzione continua. È un termine ferito, come l'aurora che contiene e guarda.

Tuttavia, lo afferma spesso e con convinzione, per Zambrano, la democrazia, integrata con la costellazione della parola persona e intesa come spazio e luogo in cui «no sólo es permitido, sino exigido, el ser persona", ${ }^{24}$ è l'unico cammino da perseguire. È una via più musicale che architettonica, perché ciò che la caratterizza in modo precipuo è che deve essere sempre creata.

2I. «No hay crisis, lo que hay más que nunca es orfandad». "Al tempo dell'edizione originaria dell'opera -scrive Bombacci - era plausibile parlare di una crisi dell'uomo europeo e, in prospettiva più ampia, occidentale. Adesso, anche se la storia continua a costruire idoli e a richiedere vittime e sacrifici, la sua struttura sacrificale non è più abbastanza manifesta. All'autrice non sembra più possibile parlare di crisi bensì di un abbandono più forte che mai.»«Persona $e$ democrazia nel pensiero di María Zambrano", in Dialegesthai 2016, url: https://mondodomani.org/dialegesthai/ nbio.htm.

22. Zambrano, M., «Prólogo», in Persona $y$ democracia, cit. p. II.

23. Ibidem.

24. Zambrano, M., Persona y democracia, cit. p. 169 . 
25. Cfr. Zambrano, M., Persona e democrazia. La storia sacrificale, trad. it. Claudia Marseguerra, Milano, Bruno

Mondadori, 200o, p. 2.

26. Zambrano, M., Verso un sapere dell'anima, edizione italiana a cura di Rosella Prezzo, Milano, Raffaello Cortina Editore, 1996, p. 8I.

27. Potente, A., «Ricostruire senza fondi: misticopolitica della creatività femminista", in Femminismo fuori sesto. Un movimento che non può fermarsi, pp. 95-IO2, cit. p. 98.

28. Zambrano, M., "Prólogo», in Persona $y$ democracia, cit. p I2.
Questo cammino mette allo scoperto la struttura sacrificale della storia, contraddistinta, nel momento in cui scrive il Prologo, da «orfandad». La crisi non esiste quasi più, ora c'è una percezione e un senso e uno stato di abbandono più forte che mai. Nella traduzione italiana di Persona y democracia, "orfandad» viene tradotta con «senso di abbandono». ${ }^{25}$

Non si è più propriamente in crisi, ma si vive uno stato di abbandono. Sono due cose distinte.

La crisi, «ogni crisi storica ci rivela chiaramente un conflitto essenziale della vita umana», perché attraverso la crisi abbiamo «il privilegio di potere vedere chiaramente la nostra vita, come se fosse allo scoperto grazie a se stessa e non per merito nostro». ${ }^{26}$ In questo senso, la crisi è sempre epifanica. Nello scoprire e rivelare la nostra vita, la crisi ci fa vivere inquieti e confusi. Un'inquietudine «determinata o eccessiva», ma in ogni caso estrema, in quanto mostra la nuda vita. Ed è davanti a questa nudità che occorre evitare l'astrazione di una risposta consolatoria e priva di responsabilità. La vita in crisi spinge a ricercare una forma di vita, "una figura della realtà» nella quale la nostra esistenza abbia un senso. Ciò a cui invita l'epifania della crisi è a non coprirsi in qualsiasi modo, "con qualsiasi cosa». È il rischio della vita in crisi che, mostrandosi nelle sue viscere, può anche reificarsi in se stessa o rimanere senza guida e appigli. Ma se non si fugge, se non c'è ansia di fare storia a tutti i costi, se non c'è indifferenza e alienazione, la crisi invita ad esserci, «con affetto del presente e non abbandono delle situazioni» ${ }^{27}$ usando un' espressione di Antonietta Potente.

Se è vero che anche nella crisi possono esserci momenti di solitudine, si tratta di una solitudine diversa da quella vissuta nella «orfandad». La parola orfanità è connessa in senso etimologico con il termine latino orbus, che significa privo, mancante. Orbo significa anche cieco, privo di vista.

Questa condizione di privazione, di abbandono è descritta così:

Hoy no se ve ya el sacrificio: la historia se nos ha tornado en un lugar indiferente donde cualquier acontecimiento puede tener lugar con la misma vigencia y los mismos derechos que un Dios absoluto que no permite la más leve discusión. Todo está salvado y a la par vemos que todo está destruido o en vísperas de destruirse. [...] Mostrarlo requeriría superponer una meditación entrecruzada y, especialmente, la reaparición de la memoria perdida. ${ }^{28}$

La "orfandad», nella storia che ora è un luogo di indifferenza, dove ciò che avviene non è altro che la successione fattuale di accadimenti subiti passivamente, è lo stato di chi non vede e neppure è visto. Indifferenza, passività, mancanza di visione caratterizzano l'orfanità della creatura umana. 
E poiché la condizione umana è per Zambrano segnata dal nascersi e rinascere continuo, dal «bisogno di darsi alla luce», di vedersi e di essere visto, ${ }^{29}$ "ahora es cuestión de volver a nacer» e "hay que esperar, sí, o más bien, no hay que desesperar [...] que se repita el fiat lux». ${ }^{30}$ Per questo Persona y democracia, introdotta dal Prologo, è testimonianza di coscienza storica e attenzione al tempo opportuno di dire parole di verità attraverso un pensiero meditato e una memoria recuperata. Il Prologo, oltre che un intertempo, è quindi evidenza propria di una confessione e guida. Entrambe, confessione ed evidenza, "sono generi intermedi fra due estremi: da un lato la superbia e il mascheramento, dall'altro un certo compiacimento della dispersione e del capriccio, un certo narcisismo». ${ }^{3 \mathrm{I}}$

\section{Una costellazione semantica}

«Pues las palabras se reúnen y agrupan en constelaciones como los astros, pero más movibles que ellos se separan y entran en relación con otras de las que estuvieran separadas»..$^{2}$

Tra le parole che si uniscono formando una costellazione ce ne sono due significative: coscienza, aggettivata come storica e tempo dell'istante: tempo che precede e invita al risveglio e che obbliga la coscienza a prendere forma, attraverso la perplessità e la confusione.

Per Zambrano, l'essere umano è un essere storico e pertanto dotato di coscienza storica. Ma questa coscienza oltre ad essere storica è anche generazionale e quindi genealogica. La genealogia, quando si mostra, mostra il mondo. Generando la propria storia, l'essere umano «guarda oltre se stesso. La storia non nasce da un uomo solo, ma deve avere una tradizione o qualcosa che la provochi, nasce con una determinazione, in una circostanza». ${ }^{33} \mathrm{Da}$ qui l'importanza della memoria, del tempo, come continuità che passa trasformandosi, e della convivenza: «sabemos que convivimos con todos los que aquí viven y aun con los que vivieron». ${ }^{34}$

Ci sono tre modi in cui la coscienza storica-generazionale-genealogica agisce.

Attraverso di essa, innanzitutto, si asseconda ciò che chiede la speranza e reclama la necessità. È il momento in cui la speranza abbraccia la necessità, senza rimanervi impigliata. La coscienza storica riesce a non abbandonare la speranza, che altrimenti delira, e a soddisfare la necessità impedendole di costruire incubi. Per questo la coscienza storica è il contrario della rivoluzione, non agisce immediatamente, nel tentativo di liberarsi da un incubo, bensì è un processo segnato da confusione e perplessità che, insieme, costituiscono gli antecedenti vitali della presa di coscienza. Non tutto subito, non tutto ora, ma senza venire meno al fondamento della necessità che è sempre urgente e immediato. Unire l'urgenza della necessità con la speranza, che invece ha un contenuto e un argomento che
29. «Non ci sarebbe storia [...] se l'uomo non fosse questa creatura che ha bisogno [...] di vedersi e di essere visto [...] Bisognoso di darsi alla luce [...] di continuare a nascersi», María Zambrano, «L'esperienza della storia (dopo di allora) [1977]", in Aut Aut, Milano, maggiogiugno 1997 , p. I4.

30. Zambrano, M., «Prólogo», in Persona $y$ democracia, cit. p I2.

3I. Prezzo, R., «Introduzione all'edizione italiana. Il Cominciamento", in Verso un sapere dell'anima, pp. VII-XXIV, cit. p. XXIV.

32. Zambrano, M., Persona y democracia, cit. p. I7I.

33. Zambrano, M., Delirio e destino, edizione completa rivista da Rogelio Blanco Martínez e Jesús Moreno Sanz, edizione italiana a cura di Rosella Prezzo, Milano, Raffaello Cortina Editore, 200o, p. 196.

34. Zambrano, M., Persona y democracia, cit. p. 25 . 
35. Cfr. Zambrano, M., «De la necesidad y de la esperanza", in Filosofía y Educación. Manuscritos, edición de Ángel Casado y Juana Sánchez-Gey, Málaga, Editorial Ágora, 2007, pp. I23-I28.

36. Zambrano, M., Persona y democracia, cit. p. 24.

37. Ivi, cit. p. 26. sostanzia la vita, senza una compiutezza immediata, è l'equilibrio ricercato per una ermeneutica della storia e non per la sua costruzione a tutti i costi. ${ }^{35}$

La coscienza, attraverso la confusione e la perplessità, permette la "historia verdadera» che è giocata tra passato e "porvenir». Solo passato e "porvenir "possono riscattare la storia e renderla vera, solo a patto che ciò che sta per venire non sia la ripetizione delle condanne e degli errori del passato.

Inoltre, la caratteristica a mio avviso più significativa della coscienza storica è il fatto di tenere in considerazione,

[...] y aun el pretender abarcar los acontecimientos todos que se registran en cualquier parte del planeta: el que el hombre de hoy viva la historia universal en sentido horizontal; también, diríamos, el que sintamos ligados entre sí como partes de un mismo drama los sucesos ocurridos en los lugares más alejados del país en que vivimos. ${ }^{36}$

Questa presa di coscienza nasce sia dalla condizione di esilio vissuta, dove è forte la dimensione pratica e simbolica di appartenere al mondo, senza vincoli di patria, sia da quella capacità di ri-significare il termine pietas secondo una accezione simile all'empatia di Edith Stein. Questa presa di coscienza dovrebbe, inoltre, in una società come la nostra, che sembra essere ormai anestetizzata di fronte al dramma e alla tragicità di certi eventi, diventare un richiamo costante e un appello a non sentire «nuestra persona como una fortaleza en cuyo interior estamos encerrados». ${ }^{37}$ 